\title{
Empirical Evaluation of Superposition Coded Multicasting for Scalable Video
}

\author{
Chun Pong Lau*, Basem Shihada*, and Pin-Han $\mathrm{Ho}^{\dagger}$ \\ *Computer, Electrical and Mathematical Science and Engineering Division \\ King Abdullah University of Science and Technology, Thuwal 23955-6900, Saudi Arabia \\ \{lau.pong, basem.shihada\}@kaust.edu.sa \\ ${ }^{\dagger}$ Department of Electrical and Computer Engineering \\ University of Waterloo, Waterloo, Ontario, Canada N2L $3 G 1$ \\ p4ho@uwaterloo.ca
}

\begin{abstract}
In this paper we investigate cross-layer superposition coded multicast (SCM). Previous studies have proven its effectiveness in exploiting better channel capacity and service granularities via both analytical and simulation approaches. However, it has never been practically implemented using a commercial 4G sytem. This paper demonstrates our prototype in achieving the SCM using a standard 802.16 based testbed for scalable video transmissions. In particular, to implement the superposition coded (SPC) modulation, we take advantage a novel software approach, namely logical SPC (L-SPC), which aims to mimic the physical layer superposition coded modulation. The emulation results show improved throughput comparing with generic multicast method.
\end{abstract}

Keywords-Superposition Coding, Scalable Video Coding, Wireless multicast, broadcast, Cross-layer

\section{INTRODUCTION}

The emerging wireless broadband technologies, such as Long Term Evolution Advanced (LTE-Advanced) and Worldwide Interoperability for Microwave Access (WiMAX), enable quadruple-play services (i.e., voice, data, video, and mobility), which are expected to push the revenue of the Internet service providers. Cisco forecasts that video will be the dominating and bandwidth-intensive traffic in the future wireless networks [1]. To support video multicast/broadcast services, a base station (BS) may serve several hundred users at the same time with different channel conditions and display capabilities, leading to a multi-user channel diversity problem. This makes it challenging for the BS to determine an effective multicast transmission scheme for scalable and high-quality video multicasting.

For example, different subscribers have different Signalto-Noise Ratio (SNR) according to their locations and perceived interferences. Users with good channel conditions can accept a high transmission rate. However, the users with bad channel conditions can only accept lower speed transmissions. It is critical to find an effective and scalable transmission scheme that can create finer granularity in the modulation at the $\mathrm{BS}$ and demodulation at the group of receivers with channel diversity.

Recent schemes introduced in the literature, propose using scalable and robust video multicast/broadcast services in wireless networks. [2] proposed a 2-level forward error correction (FEC) mechanism to recover lost video data. A cooperative error recovery approach was presented in [3] by a rate selection algorithm within a 2-phase cooperative framework. Stimulate cooperation was presented in [4] in which some of the receivers nodes are designated to redistribute the data transmitted by the BS. These schemes were evaluated using mathematical modeling and simulation without any empirical evaluation. The work of [5] presented the first real implementation of streaming video data on fixed WiMAX by using an empirical emulated testbed. Yet it only considered a generic scenario where the video bitstreams are transmitted in series without any further manipulation.

Our previous works in [6], [7] proposed a cross-layer SCM framework to tackle the multi-user channel diversity issue, achieving flexible and fine-granularity delivery of scalable video data via both simulation and analytical modeling. However, the use of SPC requires physical layer superposition of two analog signals and is not supported by the current commercially available BSs. This fact makes the approach in [6], [7] not practically realizable. In addition, the lack of experiment at results using a real $4 \mathrm{G}$ BS and receiver has not yet been evaluated.

This paper aims to examine the SCM scheme and observe the possible performance improvement by using an emulator based on NI 5641R that runs the standard IEEE 802.16e protocol stack. To mimic the superposition of two analog signals, we employ the technique in [8] - a software approach that can mimic the effect of SPC of two signals modulated separately. The emulation results validate the approach in [8] and verify the advantages of using SCM claimed in [6] and [7] by comparing it with generic multicast/broadcast schemes reported in [5] in terms of video quality.

The rest of this paper is organized as follows. Section II presents the related background, and the advantages of SCM. Section III presents the employed strategy for software implementation of the SCM. System architecture of the emulator-based implementations and other design components are presented in Section IV. Section V discusses the empirical results. Finally, Section VI concludes the paper. 


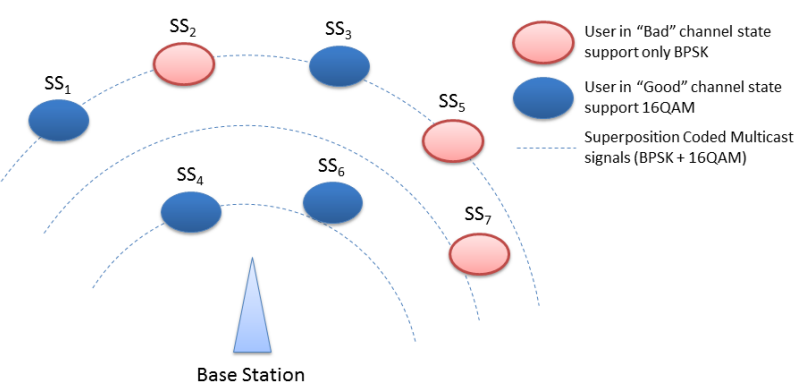

Figure 1: A single common multicast signal (either using BPSK, 16QAM or SCM) from a single base station

\section{BACKGROUND}

\section{A. Multi-user Channel Diversity Problem}

Consider the scenario that multiple users are in the coverage of a single BS as shown in Fig. 1. These users experience different channel conditions. Users with good channel can afford to receive data from the BS using an aggressive modulation such as 16-QAM. On the other hand, users with bad channels can only manage conservative modulation such as BPSK. If a conservative modulation is used to multicast a low bitrate basic quality video, all users can then receive it robustly. However, this underutilizes the channel capacity of users with good channel conditions. On the other hand, if an aggressive modulation scheme is employed, less resources are consumed at the BS but users with bad channel conditions cannot decode received signals. This scenario highlights the challenges in using multicasting/broadcasting service in wireless network. A strategic transmission scheme is therefore desired to develop and implement wireless video multicast/broadcast in BS.

\section{B. Scalable Video Coding}

Scalable Video Coding (SVC) is extended from H.264/AVC [9]. Its most generic format provides a layered video bitstream that consists of data in the base layer and one/more enhancement layer. A SVC decoder can decode a received video with only the base layer bitstream, while any additional enhancement layer data received can be used to refine the video quality.

There are three types of scalability improvement in SVC named temporal, spatial, and quality. Temporal scalability provides a frame rate scalability with some enhancement layers to increase the number of frames per second of a video. Spatial scalability provides different picture sizes of the video. The lower spatial resolution layers can be used to predict the higher spatial resolution layer for reducing the bitrate. Quality scalability refers to the SNR of the picture, improving the visual quality of a video with enhancement layer. The work in [9] explains the three scalability schemes.

The encoded video bitstreams are structured with network abstraction layer (NAL) units. Each picture data is encapsulated into one NAL unit. The header of each NAL unit indicates the type (i.e., base or enhancement layer) of contained data. The remaining bytes of the units are the payload. If base layer NAL units are received correctly, a base quality video can be played back. If enhancement NAL units are received correctly, the video quality can be improved.

\section{Superposition Coding}

Superposition coding (SPC) can transmit independent symbols simultaneously by superimposing them into one multi-resolution modulated wireless signal. Each of these multi-resolution modulated signals is decodable by multiple users under different channel conditions. The superposition of two symbols can be explained as the vector addition of the two signal constellations. Authors in [10] proved that using SPC strategically in downlink CDMA network can increase the channel capacity.

\section{2-level Superposition Coded Multicast}

2-level SCM was proposed in [6], [7] for achieving wireless video transmissions by superimposing successive refined source symbols from scalable video bitstreams. In the terminology of two-layer scalable video coded bitstreams, each SCM signal contains the symbol corresponding to some bitstream bits for base video quality layer modulated by a lower-order modulation scheme, which is superimposed with another symbol of the corresponding video data in the enhancement quality layer modulated by a higher-order modulation scheme. A multicast receiver with a good channel can decode the whole SPC signal via signal-interference cancellation (SIC) and obtain both quality layers from the superimposed multicast signal. On the other hand, the receiver with bad channel quality can mostly decode the base layer information modulated by a lower-order modulation scheme and obtain the base quality layer. The advantage of SCM over the conventional mono modulated multicast strategy is to create finer granularity of signal qualities so as to achieve a scalable transmission under varying channel condition. Its advantage has been well proved in [6], [7] by way of analysis and simulation. Fig.2 illustrates the SCM architecture via conventional SPC modulation and SIC based demodulation [6].

\section{SOFTWARE IMPLEMENTATION OF SCM}

This section presents our previously reported software implementation approach [8], namely logical SPC (L-SPC), that can mimic the superposition of two digital signals modulated separately.

\section{A. One-shot Modulation at Transmitter}

The goal of the L-SPC transmitter is to mimic SPC symbols generated by conventional analog SPC, which nonetheless requires dedicated hardware for superimposing 


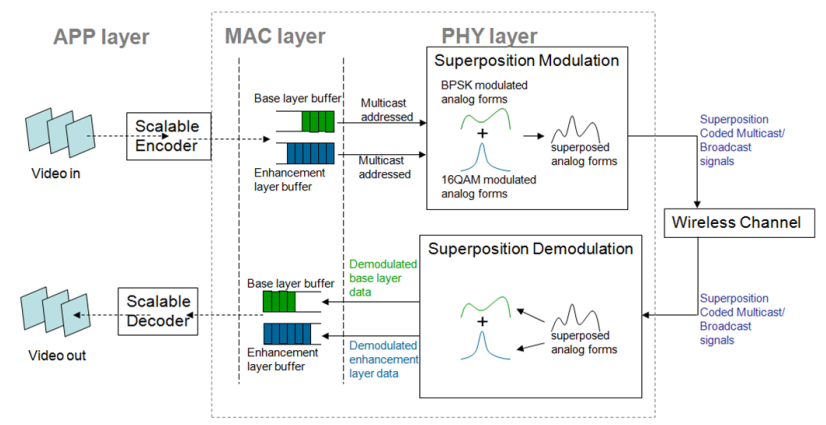

Figure 2: The SCM architecture [6]

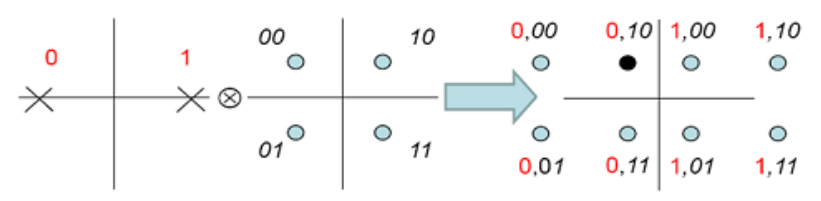

Figure 3: Mapping a 3-bit symbol to one of the eight constellation symbols

two modulated signals. A superimposed signal can be taken as the summation of the two vectors expressed in terms of the corresponding amplitudes and phases in a constellation diagram, formed by the conventional approach using $m_{1}$ QAM and $m_{2}$-QAM, where $m_{2}>m_{1}$. Note that BPSK is treated by a 2-QAM modulation scheme for the generalization purpose. The resultant constellation diagram of signal consists of $\mathrm{m} 1 \mathrm{~m} 2$ points, each with a unique amplitudephase combination determined by the allocation ratio of energies for the $m_{1}$-QAM and $m_{2}$-QAM signals, denoted by $\mathrm{E} 1$ and $\mathrm{E} 2$, respectively. A total energy constraint exists, $E=E_{1}+E_{2}$, with $\beta$ as the single parameter governing the relationship between $E_{1}$ and $E_{2}$ :

$$
\begin{gathered}
E_{1}=\beta E \\
E_{2}=(1-\beta) E
\end{gathered}
$$

By identifying the required number of constellation points and manipulating the $\beta$ value for energy allocation, the proposed L-SPC mimics the conventional SPC signal based on $m_{1}$-QAM and $m_{2}$-QAM signals for the base and enhancement layer source bitstreams, respectively, using a standard $m_{1} m_{2}$-QAM signal. As a result, the conventional SPC for superimposing two modulation signals of an arbitrary $\beta$ value, can be simply performed by a one-shot modulator where the amplitude and phase are manipulated corresponding to $\beta$. The mechanisms necessary in achieving amplitude and phase assignment are already available in commercially available modern wireless chipsets [11], thus eliminating any major hardware or software modifications by replacing the conventional SPC modulator with the proposed one-shot modulator.

\section{B. A Cross-layer Mapping at Transmitter}

To realize the proposed L-SPC modulation for video multicast, strategic mapping a symbol of $\log _{2} m_{1}$ bits from the base layer data and $\log _{2} m_{2}$ bits from the enhancement layer into a $\left(\log _{2} m_{1} m_{2}\right)$-bit symbol block is required. Fig. 3 demonstrates a case study for $m_{1}=2$ and $m_{2}=4$, in which each symbol block contains one bit from the base layer and two bits from the enhancement layer, corresponding to the number of bits in the respective 2-QAM (BPSK) and 4QAM (QPSK) symbol. The left portion of Fig. 3 illustrates the summation of two conventional modulation schemes to yield the resultant constellation diagram with eight points, as shown in the right portion of Fig. 3, mapped from the 3bit symbol. For a base layer symbol ' 0 ' and a enhancement layer symbol ' 01 ', their concatenation ' 0,01 ' can be mapped to ' 001 ' in the one-shot SPC constellation diagram to generate a logical SPC modulated signal equivalent to the conventional approach.

\section{One-Shot Demodulation at Receiver}

In an effort to accommodate hardware constraints, LSPC leverages existing receiver demodulators to eliminate the hardware-based SIC process for demodulating SPC signals. The proposed L-SPC demodulation decodes data of both layers using a standard $m_{1} m_{2}$-QAM demodulator. The first $\log _{2} m_{1}$ bits corresponding to the base layer data will be subject to a lower error rate, where an equal base layer symbol error rate in comparison with that by the conventional SIC approach can be achieved by employing a strategic cross-layer mappings of information bits at the transmitter discussed in the previous subsection. The base layer decoded $m_{1} m_{2}$-QAM symbol is always strategically mapped to differentiate between the $m_{1}$-QAM decision regions, which are the equivalent $m_{1}$-QAM decision regions utilized by the SIC demodulation process in conventional SPC. Hence, both L-SPC and conventional SPC result in the same base layer symbol error. The enhancement layer data, on the other hand, will also be taken from the oneshot demodulation with a larger error rate than that by the conventional SIC-based approach. However, due to the successively refined characteristic of the base and enhancement layers, the impairment on the overall performance is very limited. The corresponding analysis can be found in [8]

The simplification of the SPC symbol decoding process, however, requires support in the software to handle the decoded video information bits for playback, which is discussed below.

\section{Required Software Support at Transmitter and Receiver}

The implementation of L-SPC modulation at the transmitter and demodulation at receivers needs a new software module requiring the knowledge of information bits dependency between the two quality layers in a scalable video source. Information bits from different layers are buffered 
in the corresponding queues at the transmitter. A set of software primitives facilitate the interaction between a modified medium access control (MAC) layer and the physical (PHY) layers for the software support to execute an oneshot modulation scheme. An interaction in the implemented software selects a constellation point to map the set of bits at the head-of-line from the corresponding queues to a 3-bit symbol. In the modulation chipset, more functions should be added such that service access points (SAPs) are defined to recognize parameters passed from the MAC software in a BS for this one-shot modulation process.

With knowledge of the two modulation schemes employed at the transmitter, minor software modifications is needed at the video source in splitting information bits in each decoded symbol for the base and enhancement layers. The final necessary mechanism is to extract bits from both buffers for the video decoder in a receiver to reconstruct the video. Similar to the conventional SPC, minimal signaling is necessary for L-SPC since both transmitter and receivers need to synchronize the choices of two modulation schemes selected for transmission. In addition, the chipset should generate the L-SPC modulated signals according to the strategic energy allocated for each modulation due to the required mapping of a constellation point. Since variable energy allocations and dynamic phase keying assignments are becoming common in modern wireless chipsets, the aforementioned software modifications do not introduce impractical overheads to the state-of-the-art BS system.

In comparing L-SPC with the conventional SPC demodulation process, both schemes need separated buffers and interfaces to handle the individual streams of information bits from the multi-stage decoding process. However, the modified software in the L-SPC scheme yields all information bits from a single demodulation process in one-shot, and then assigns the first $\log _{2} m_{1}$ bits and subsequent $\log _{2} m_{2}$ bits into buffers of the base and enhancement layers, respectively, which is a relatively lightweight procedure.

\section{EXPERIMENTAL SETUP}

\section{A. Overview of System Hardware}

Emulation gives the flexibility for easy configuration for evaluating various changes in system design. This can also provide an empirical insight for future development such as implementing a chipset.

Our emulator is constructed mainly in three logical layers: Application (APP), MAC and PHY layers. APP is implemented in the computer connected to the emulator, where the scalable video source is first separated into two quality layers, namely base and enhancement. The separated bitstreams are then transmitted into two buffers and fed into the MAC of the emulator, which is further divided into two parts: MAC Core and MAC Convergence Sub-layer (MAC CS). These two sub-layers communicate using Real-Time FIFOs. The PHY layer of the transmitter includes the SAP

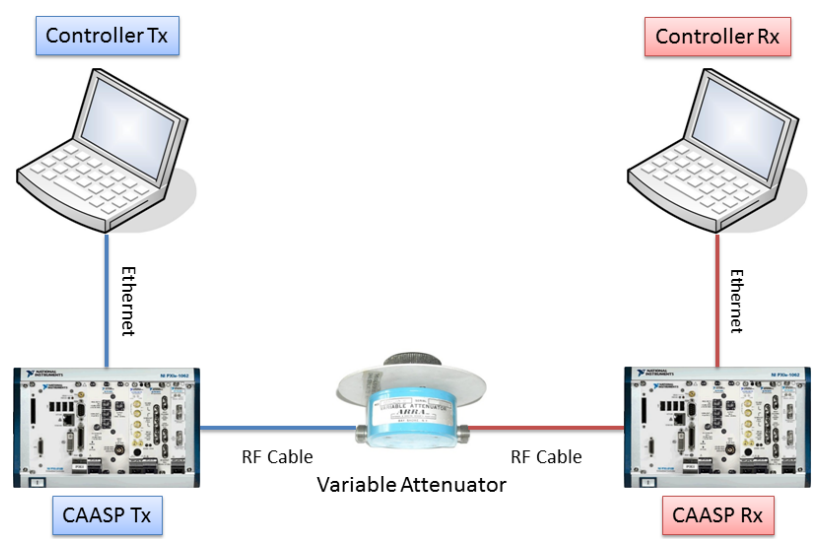

Figure 4: Experimental Setup

Interface, FEC unit, and the OFDMA Processor. The receiver has the same blocks as transmitter, which is synchronized with the transmitter via a synchronization unit.

Fig. 2 shows the system architecture of three layers. APP is implemented on a Windows computer. MAC is designed in LabVIEW Real-Time operating system and it is implemented by using the Embedded Real-Time PC Controller NI PXIe-8130 of the emulators. PHY is designed using VHDL and LabVIEW FPGA and it is implemented by the base-band FPGA-base processing board namely NI PXIe-5641R [12].

Experiments were conducted on the emulation testbed for implementing the 2-level SCM. Fig. 4 shows the testbed overview, the Base-broadcasting Station (BS) and Mobilesubscribing Station (MS) is emulated by Controller Tx and Controller Rx, respectively. Two emulators are named as CAASP Tx and CAASP Rx.

The testbed has two main components: a video section and a wireless emulation section.

The video section consist of a BS and a MS. Both BS and MS are emulated by two separate computers. The CAASP Tx broadcasts an SVC bitstream through the wireless network to the CAASP Rx for decoding. The received video is played back and store for post analysis. A raw video 'Sintel' [13] is encoded by JSVM 9.18 encoder from Heinrich-HertzInstitute into a two-layer SVC video with standard definition $(864 \times 480 p)$. The video has 1242 fames in total with the rate at 30 frames per second and other properties are listed in Table II.

On the other hand, the wireless emulation section consists of CAASP Tx and CAASP Rx manufactured by National Instruments. Downlink (DL) protocol is implemented over a $2.51 \mathrm{GHz} 802.16 \mathrm{e}-2005$ standard system. The CAASP-Tx consists of the IF-RF Up Converter (NI PXI-5610), the FPGA-base baseband processing board (NI PXIe-5641R) and the Real-time Controller (NI PXIe-8130). The CAASPRx has similar components, but the IF-RF Up Converter is replaced by a RF-IF Down Converter (NI PXI-5600). 

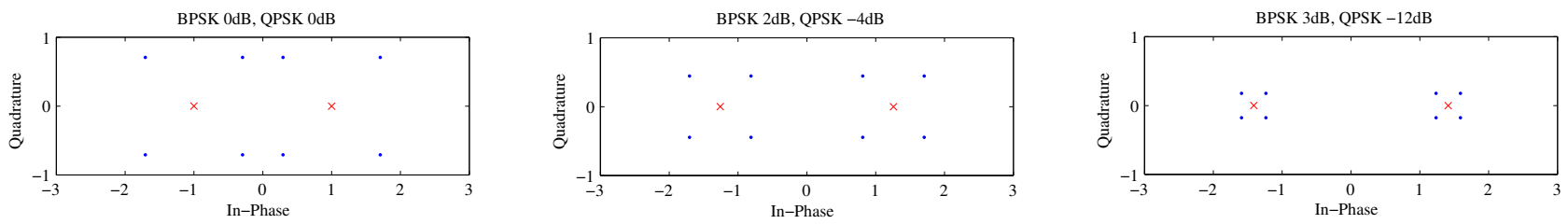

Figure 5: Constellation of three power allocation schemes

Table I: Network Characteristics

\begin{tabular}{|l|c|c|}
\hline Transmission type & SCM & Generic Multicasting \\
\hline Air Interface & IEEE802.16e-2005 DL only \\
\hline Duplex mode & \multicolumn{2}{|c|}{ TDD } \\
\hline FFT Size & \multicolumn{2}{|c|}{1024 Bytes } \\
\hline Nominal Bandwidth & \multicolumn{2}{|c|}{$10 \mathrm{MHz}$} \\
\hline Cyclic Prefix & \multicolumn{2}{|c|}{ ms } \\
\hline Frame length & BPSK 1/2 & 16QAM 1/2 \\
\hline Base Layer Modulation & $-1 \mathrm{~dB}$ & N/A \\
\hline Base Layer Boosting & QPSK 1/2 & N/A \\
\hline Enhancement Modulation & $-5 \mathrm{~dB}$ & $2.51 \mathrm{GHz}$ \\
\hline Enhancement Boosting & \multicolumn{2}{|c|}{ 0dBm } \\
\hline Radio Frequency & \multicolumn{2}{|c|}{ 0dBm } \\
\hline Transmission Power & \multicolumn{2}{|c|}{} \\
\hline Reception Power Sensitivity & \multicolumn{2}{|c|}{} \\
\hline
\end{tabular}

CAASP Tx and CAASP Rx are connected to a variable attenuator via two $3 \mathrm{ft}$ and $6 \mathrm{ft}$ length coaxial radio frequency cables (CBL-3FT-SMSM+, CBL-6FT-SMSM+ manufactured by Mini-Circuits). These cables introduce $1.0 \mathrm{~dB}$ and $2.0 \mathrm{~dB}$ insertion loss for $3 \mathrm{ft}$ and $6 \mathrm{ft}$ respectively when the signal is transmitted in $2.5-6 \mathrm{GHz}$. The variable attenuator (4674-30FS manufactured by ARRA) aims to emulate a number of constant large scale fading wireless channel conditions with predefined signal attenuation. The reason of using close circuit instead of antennas is to prevent interference from surrounding wireless signal and provide repeatability of the experiment. Attenuation for a given receiver is kept constant during the experiments. The Controller Tx and Controller Rx are connected to the emulators by Ethernet 100BASE-TX respectively. Table I indicates the network characteristic of the system.

\section{B. SCM Signal and Power Allocation}

The SCM superimposed signal is generated by the baseband FPGA-base processing board NI PXIe-5641R in the CAASP Tx and CAASP Rx. BPSK, QPSK, 16QAM and 64QAM are four modulation schemes available in the emulation, which is performed at the OFDMA Processor. The transmission power between base and enhancement layer can be dynamically adjusted. Fig. 5 shows the constellation diagram of an example that superimposes a BPSK signal with a QPSK signal with different power allocation schemes.

\section{EMUlation Result}

Five samples were collected for each attenuation value while varying the total attenuation between the transmitter
Table II: Video Encoding Properties

\begin{tabular}{|l|c|c|}
\hline Number of Frames & \multicolumn{2}{|c|}{1242 frames } \\
\hline Frames Rate & \multicolumn{2}{|c|}{$30 \mathrm{fps}$} \\
\hline Resolution & \multicolumn{2}{|c|}{$864 \times 480$ pixels } \\
\hline Group of Picture & \multicolumn{2}{|c|}{4} \\
\hline Intra Period & \multicolumn{2}{|c|}{ CAVLC } \\
\hline Symbol mode & \multicolumn{2}{|c|}{ Yes } \\
\hline Inter-layer Prediction & Base & Enhancement \\
\hline Layer & 35 & 25 \\
\hline Quantization Parameters & 521.63 & 1686.88 \\
\hline Bitrate(kbps) & 56.01 & 59.12 \\
\hline Encoding PSNR(dB) & BPSK 1/2 & QPSK $1 / 2$ \\
\hline Correlated SCM Modulation & \multicolumn{2}{|c|}{$16 \mathrm{QAM} 1 / 2$} \\
\hline Correlated Generic Multicast Modulation & \multicolumn{2}{|c|}{} \\
\hline
\end{tabular}

and receiver from $5 \mathrm{~dB}$ to $12 \mathrm{~dB}$ in $\mathrm{GM}$ and $5 \mathrm{~dB}$ to $15 \mathrm{~dB}$ in SCM. A total of 95 data samples were collected. We implemented the SCM scheme on the developed emulator, and the result was further compared with that by a generic scheme in [5], namely GM. The performance metrics considered in the evaluation are Base layer Packet Error and Loss Rate (BPELR), Enhancement layer Packet Error and Loss Rate (EPELR) and the Peak Signal-to-Noise Ratio (PSNR). PSNR is a full-reference objective video quality metric to measure the error after video compression and network transmission. Higher video quality at the receiver result in a higher PSNR value. The results are presented in Fig. 6. Note that in the emulation each packet is considered as a sub-layer frame of scalable video. If an enhancement layer packet is corrupted, lost or truncated, the corresponding base layer packet would be used for decoding, which results in a degraded quality of that picture frame.

The emulation results show that when the channel conditions are good (i.e. the channel attenuation is lower than 8dB), B-PELR and E-PELR are low, and the video quality measured by PSNR is close to the original video for both GM and SCM.

In case of $\mathrm{GM}$, when attenuation is $8 \mathrm{~dB}$ or higher, the E-PELR increases from $0.20 \%$ at $8 \mathrm{~dB}$ to $3.17 \%$ at $10 \mathrm{~dB}$. The PSNR dropped from $55.37 \mathrm{~dB}$ to $42.32 \mathrm{~dB}$ although the E-PELR dropped in a small amount. The PSNR is seen dropping dramatically to $26.08 \mathrm{~dB}$ with B-PELR and EPELR are $12.15 \%$ and $45.63 \%$ at $12 \mathrm{~dB}$. The video cannot be played when attenuation is greater than $12 \mathrm{~dB}$.

In case of SCM, when attenuation is smaller than $11 \mathrm{~dB}$, both B-PELR and E-PELR are closed to $1 \%$, the PSNR 


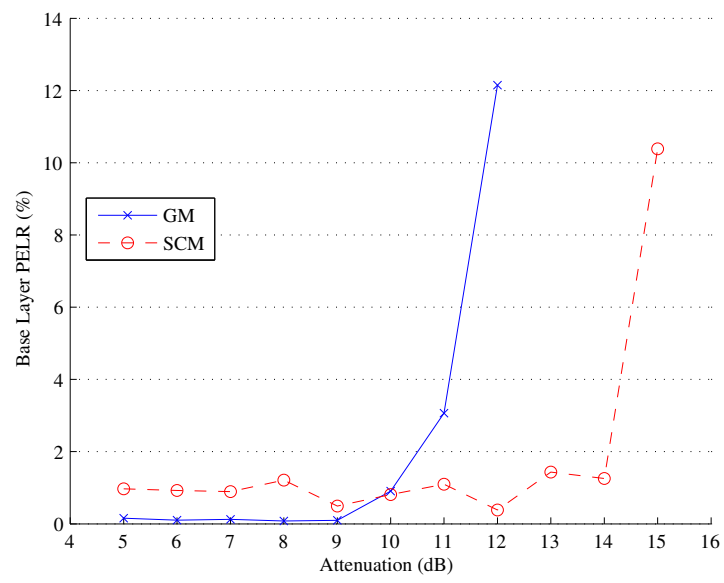

(a) Base Layer Packet Error and Loss Rate

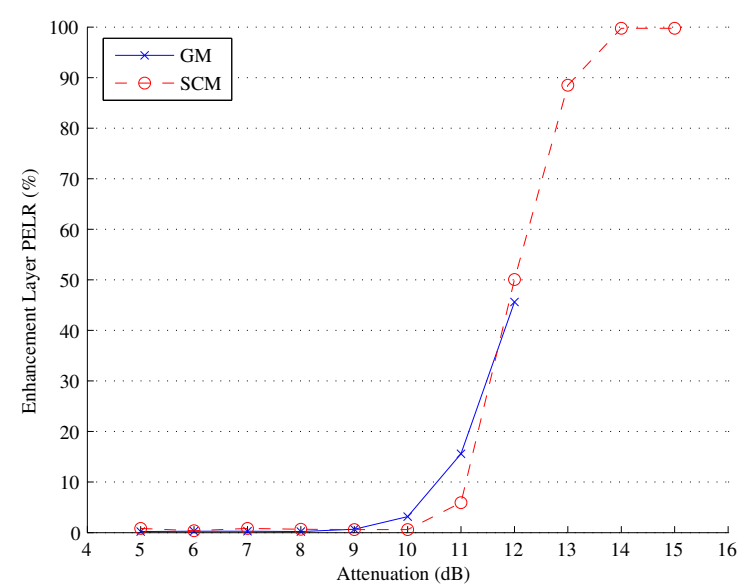

(b) Enhancement Layer Packet Error and Loss Rate

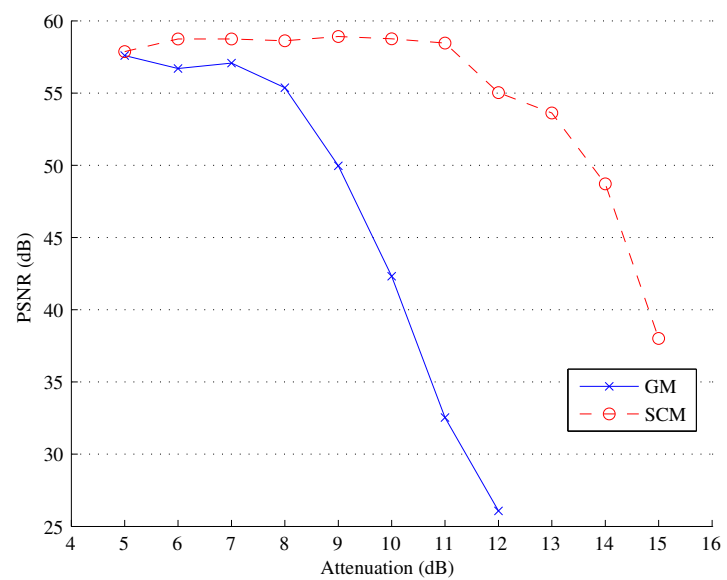

(c) PSNR

Figure 6: Experimental Result

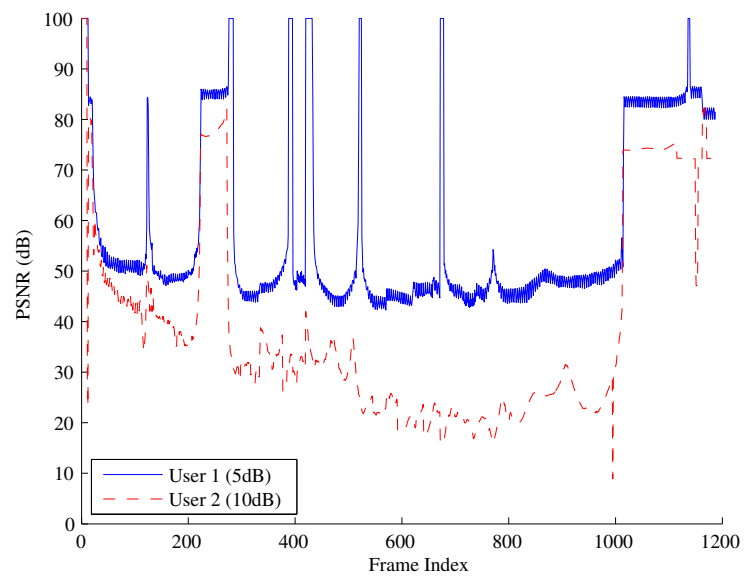

(a) Received video PSNR of users using GM

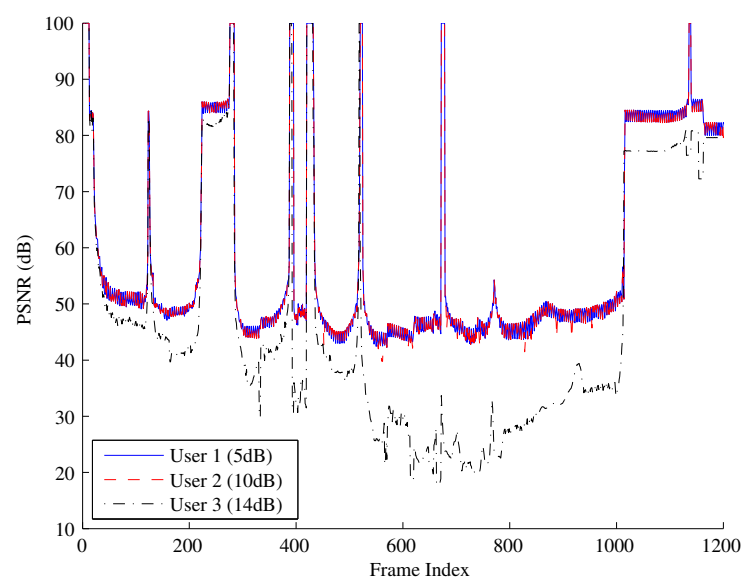

(b) Received video PSNR of users using SCM

Figure 7: PSNR of each frame in sequence

is shown good which yields a good quality of video. Note that when at $11 \mathrm{~dB}$, although E-PELR starts increasing from $0.5 \%$ to $6 \%$, PSNR can still be high and video remains in a good quality. When E-PELR reached $50 \%$ at $12 \mathrm{~dB}$, the PSNR drops significantly to $55.04 \mathrm{~dB}$; while at $14 \mathrm{~dB}$, we see that all the enhancement layer packets are lost, but most of the base layer packets can still be received with B-PELR at $1.25 \%$ such that a base video quality can be obtained with PSNR is $48.72 \mathrm{~dB}$. At $15 \mathrm{~dB}$ where B-PELR reaches $10.4 \%$, the video quality become unacceptable. When attenuation is greater than $15 \mathrm{~dB}$, most of the packets are lost, and video cannot be played back.

To further understand the inherent problems of multi-user channel diversity, three users (specific data items selected from the dataset) are considered with channel attenuation values of $U_{1}$ to $U_{3}$ are $6 \mathrm{~dB}, 10 \mathrm{~dB}$ and $14 \mathrm{~dB}$ respectively. With $\mathrm{GM}, U_{1}$ at $6 \mathrm{~dB}$ attenuation receives the video without 


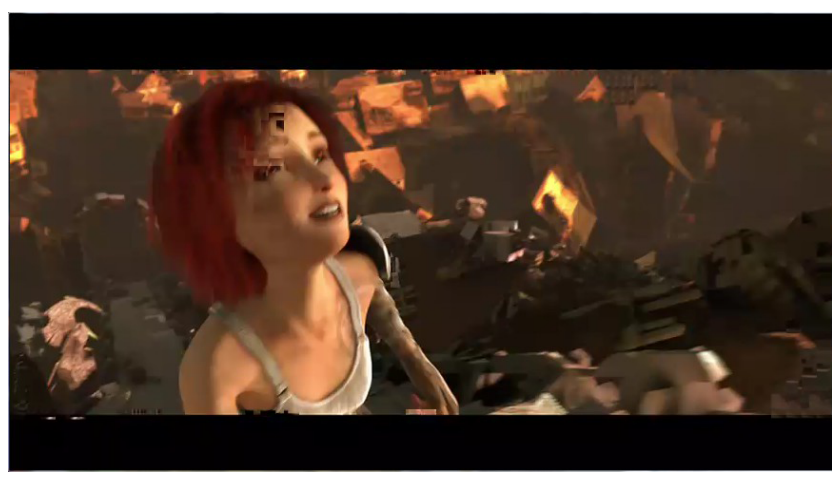

(a) Superposition-Coded Multicasting

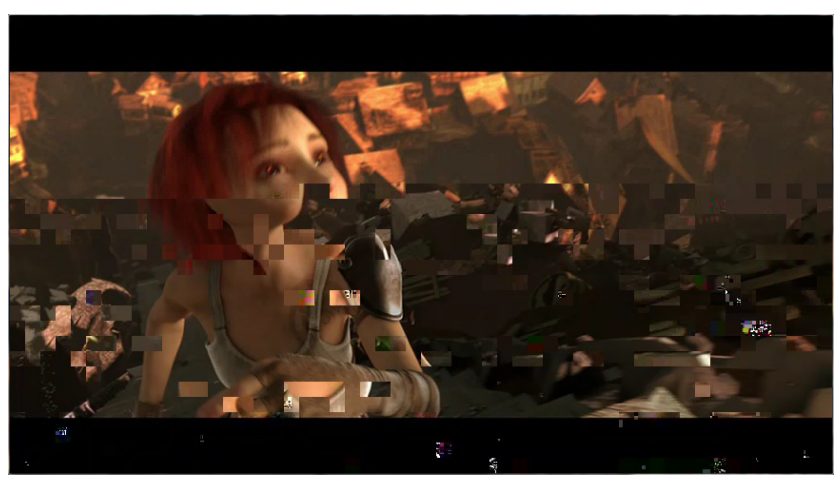

(b) Generic Multicasting

Figure 8: Screenshot of received video when attenuation at $7 \mathrm{~dB}$

any packets loss, achieving the best average PSNR 59.12dB. $U_{2}$ at $10 \mathrm{~dB}$ attenuation value suffers from the average packet loss rate of $2.49 \%$ which induce the average PSNR of $U_{2}$ by $39.17 \mathrm{~dB}$. The attenuation value of $U_{3}$ is too high where none of the packets can be successfully decoded from the signal. Therefore, $U_{3}$ cannot receive any video. The PSNR of each frame for $U_{1}$ and $U_{2}$ from beginning to the end of transmission is shown in Fig. 7a. $U_{1}$ (solid line) has a much higher average PSNR than $U_{2}$, indicating the high diversity problem between users.

SCM provides good video quality to both $U_{1}$ and $U_{2}$ at $6 \mathrm{~dB}$ and $10 \mathrm{~dB}$ with average PSNR by $58.69 \mathrm{~dB}$ and $58.45 \mathrm{~dB}$. $U_{3}$ at $14 \mathrm{~dB}$ can also receive acceptable quality of video with average PSNR 48.72dB. The PSNR of each frame for all users is shown in Fig. 7b. $U_{1}$ and $U_{2}$ achieve almost the same video quality for every frame. $U_{3}$ also receives acceptable quality video.

Fig. 8 shows the snap shots of the received video in SCM and GM over the same attenuation channel. Clearly, SCM achieves better video quality.

\section{CONCLUSION}

This paper presented our empirical experiment results on the SCM scheme, which was reported as an effective multicast strategy for successive refined sources in order to create finer granularity of video quality and better adaptation to the channel fluctuation. A novel software implementation of superposition coding (SPC) was deployed upon a commercial emulator abiding with the IEEE 802.16e standard. We successfully developed and implemented the system, and showed that using the SCM framework can significantly improve the overall quality of service against that by using a generic video broadcast scheme with mono modulation.

\section{REFERENCES}

[1] C. Index, "Global Mobile Data Traffic Forecast Update, 20092014," White Paper, CISCO Systems Inc, vol. 9, 2010.

[2] S. Zare and A. G. Rahbar, "A 2-level FEC mechanism joint with cross-layer superposition coded multicast for robust IPTV service over WiMAX," Wireless Networks, pp. 12031216, 2011.

[3] A. Azgin and Y. Altunbasak, "A Cooperative Error Recovery Framework for IPTV over WiMAX," in Consumer Communications and Networking Conference (CCNC), 2011 IEEE. IEEE, 2011, pp. 616-621.

[4] B. Niu, H. Zhao, and H. Jiang, "A Cooperation Stimulation Strategy in Wireless Multicast Networks," Signal Processing, IEEE Transactions on, vol. 59, no. 5, pp. 2355-2369, 2011.

[5] O. Issa, W. Li, and H. Liu, "Performance Evaluation of TV Over Broadband Wireless Access Networks," IEEE Transactions on Broadcasting, vol. 56, no. 2, pp. 201-210, Jun. 2010.

[6] J. She, F. Hou, P.-H. Ho, and L.-L. Xie, "IPTV over WiMAX: Key Success Factors, Challenges, and Solutions [Advances in Mobile Multimedia]," IEEE Communications Magazine, vol. 45, no. 8, pp. 87-93, 2007.

[7] J. She, X. Yu, P.-H. Ho, and E.-H. Yang, “A cross-layer design framework for robust IPTV services over IEEE 802.16 networks," IEEE Journal on Selected Areas in Communications, vol. 27, no. 2, pp. 235-245, Feb. 2009.

[8] P.-H. Ho and J. She, "System, Method, and Computer Program for Superposition Coded Multicast with a Single Modulation Scheme," U.S. Patent US 2011/0 222462 A1, Sep. 25, 2011.

[9] H. Schwarz, D. Marpe, and T. Wiegand, "Overview of the Scalable Video Coding Extension of the H.264/AVC Standard," IEEE Transactions on Circuits and Systems for Video Technology, vol. 17, no. 9, pp. 1103-1120, Sep. 2007.

[10] S. Bopping and J. M. Shea, "Superposition coding in the downlink of CDMA cellular systems," in Wireless Communications and Networking Conference, 2006. WCNC 2006. IEEE, vol. 4. IEEE, 2006, pp. 1978-1983.

[11] picoChip. [Online]. Available: http://www.picochip.com/

[12] “NI PXIe-5641R - RIO IF Transceiver,” pp. 1-3, 2012.

[13] T. Roosendaal, "Sintel," in ACM SIGGRAPH 2011 Computer Animation Festival, ser. SIGGRAPH '11. New York, NY, USA: ACM, 2011, p. 71. 